\title{
Design of distribution transformer loss meter considering the harmonic loss based on one side measurement method
}

\author{
HU Xi ${ }^{1, a}$, ZENG Jiang ${ }^{2, b}$ \\ ${ }^{1,2}$ School of Electric Power, South China University of Technology, 510080 Guangzhou, Guangdong, \\ China \\ a1054497408@qq.com, ${ }^{b} 78271793 @ q q . c o m$
}

Keywords: distribution transformer; one side measurement; harmonic loss;

\begin{abstract}
In power system, a large sum of harmonic produced by non-linear load will affect the loss of transformers. In the meanwhile, the traditional methods to measure the loss do not have enough adaptability. By analyzing the principle of one side measurement and two sides measurement methods, and making study on the harmonic copper loss and harmonic iron loss, a progressed distribution transformer loss meter considering the harmonic loss is designed in this paper. The meter with high precision, strong adaptability and operability has a wide application prospect.
\end{abstract}

\section{Introduction}

With the increase of national economy, the demand for energy is being expanded largely. As to be the main equipment of transferring voltage, current and power, transformer which has a huge sum of quantity plays an important role in power system, and the quantity is being increased by the year[1]. Despite of the little loss of each transformer, considering the large sum and the long running time, the total loss of transformers every year are surprisingly huge. In the meantime, old type transformers are still used in some areas of which the loss are much higher than the new ones. As a result, it is necessary to enhance the energy efficiency level and lower the energy loss by replacing old transformers with new ones[2,3]. So accurate measurement of transformer's loss is needed to provide basic data, in order to change transformers reasonably.

So far, most common way to measure transformer loss is traditional two sides measurement method, which is to get the loss by measuring both sides of the transformer. On one side, high measurement condition is required in this kind of measurement method. On the other side, with more and more non-linear devices being used in power system, large harmonics is produced[4], while traditional ways don't consider the loss caused by harmonics which leads to some errors. This article designed a one side transformer loss meter considering harmonic loss after analyzing the different loss of transformer.

\section{Loss Calculation Principle of Distribution Transformer}

Loss Constitution of Transformer. After the study of transformer running principle, it is found that the loss of transformer can be divided into four parts, of which the iron loss and copper loss are the main parts, almost accounts for 90 percent of the total loss. The rest are stray loss and dielectric loss. Considering the fact that the latter two kinds of losses are very little compared with the former two losses, stray loss and dielectric loss are usually neglected[5].

Magnetic hysteresis loss is produced when magnetizing velocity of iron-core material is smaller than the changing velocity of external magnetic field. And in the meanwhile, the changing induced electromotive force will generate the corresponding induced current, which make energy loss by high temperature of iron-core material. This loss is called eddy-current loss. Besides, there is still another kind of loss called added loss[6]. Magnetic hysteresis loss and eddy-current loss, which are called basic iron loss, constitute the main part of iron loss. While added loss is small enough to be ignored. So the formula of iron loss is shown in Eq. 1. 


$$
P_{F e}=P_{H}+P_{E}=K_{H} f^{2} B_{m}^{2}+K_{E} f B_{m}^{n} .
$$

$P_{H}, P_{E}$ are eddy-current loss and Magnetic hysteresis loss, $K_{H}, K_{E}$ are proportionality coefficients of two losses, $f$ is frequency, $B_{m}$ is the max magnetic flux density.

On the other side, energy loss will produced when there is current flow through resistance, which is called copper loss. In transformer, because of skin effect and proximity effect, copper loss can be divided into added copper loss and basic copper loss. Basic copper loss is regard as the energy loss caused by the high temperature of coil winding when current flow through resistance. And added loss is the extra loss caused by the increase of winding equivalent resistance[7]. The added loss has a small proportion in distribution transformer, which also can be ignored. So the copper loss shall be expressed in Eq. 2.

$$
P_{C u}=I_{1}^{2} R_{1}+I_{2}^{2} R_{2} .
$$

$I_{1}, I_{2}$ are current on both sides of transformer, $R_{1}, R_{2}$ are winding resistance of two sides of transformer.

While in practical application, the iron loss and copper loss is usually replaced with no-load loss and load loss.

Two sides measurement method. In common condition, the current and voltage of high voltage and low voltage sides will be measured to get the power of both sides, then transformer loss can be calculated by the difference of the two power value. As to the three-phase transformer.

$$
\Delta P=U_{1} I_{1} \cos \varphi_{1}-U_{2} I_{2} \cos \varphi_{2}
$$

$\Delta P$ is power loss of transformer, $U_{1}, I_{1}$ are voltage and current of primary side, $\varphi_{1}$ is phase angle of primary side, $U_{2}, I_{2}$ are voltage and current of secondary side, $\varphi_{2}$ is phase angle of secondary side.

Considering the fact that high precision measurement devices are needed in both sides of the transformer when using this traditional measurement method, which highly raise the economy costs, so a new one side measurement method is proposed in this article.

One side measurement method. According to the former analysis, transformer loss is made up by iron loss and copper loss, which can be replaced with no-load loss and load loss in practical application. So the iron loss is expressed through further derivation.

$$
\Delta P_{F e}=\frac{u^{2}}{R_{F e}}=\frac{P_{0}}{U_{N}^{2}} u^{2}=P_{0} \frac{u^{2}}{U_{N}^{2}} .
$$

$u$ is voltage, $P_{0}$ is rated no-load loss, $U_{N}$ is rated voltage.

Similarly, copper loss can be expressed as:

$$
\Delta P_{C u}=i^{2} R_{T}=\frac{P_{k}}{I_{N}^{2}} i^{2}=\frac{P_{k} U_{N}^{2}}{S_{N}^{2}} i^{2} .
$$

$i$ is current, $R_{T}$ is winding resistor, $P_{k}$ is rated load loss, $S_{N}$ is rated capacity.

Thus, the formula of transformer loss in one side measurement method can be got.

$$
\Delta P=\Delta P_{F e}+\Delta P_{C u}=\left(\frac{u}{U_{N}}\right)^{2} P_{0}+\left(\frac{i}{I_{N}}\right)^{2} P_{k} .
$$

Therefore, only one side of transformer will be measurement with one side measurement method based on the practical condition. What's more, on the condition of same precision measurement 
devices, error of one side method is smaller than two sides method, and one side method is more reasonable when put into use. As a result, this article designed a transformer loss meter based on the one side method principle.

\section{Calculation Principle of Distribution Transformer Harmonic Loss}

In recent years, with the application of power electronic equipment like SVG、STATCON、SVC, lots of harmonics produced correspondingly. And the harmonics lead to the increase of iron and copper losses. So the harmonic loss also can be divided into harmonic iron loss and harmonic copper loss. It will be analyzed below.

Principle of Harmonic Loss. Firstly, build up the t-type equivalent circuit of transformer affected by the $\mathrm{n}^{\text {th }}$ harmonics.

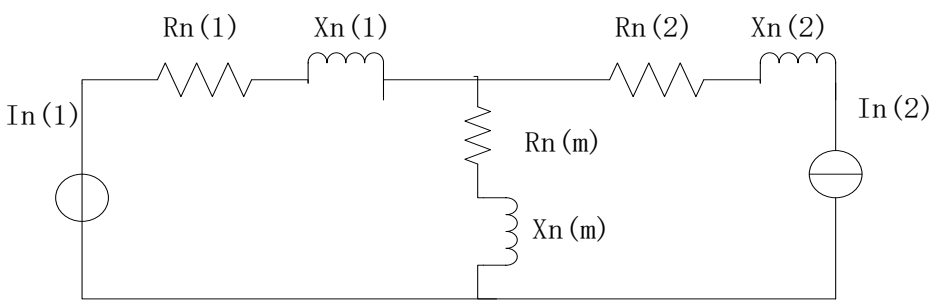

Fig. 1. T-type equivalent circuit of transformer affected by the $n^{\text {th }}$ harmonics

$\mathrm{Rn}(1), \mathrm{Xn}(1)$ are harmonic resistance and reactance of primary side, $\mathrm{Rn}(2), \mathrm{Xn}(2)$ are harmonic resistance and reactance of secondary side, $\mathrm{Rn}(\mathrm{Fe}), \mathrm{Xn}(\mathrm{Fe})$ are excitation resistance and reactance , $\operatorname{In}(1), \operatorname{In}(2)$ are nth harmonics of primary and secondary sides.

Under the effect of harmonics, the RMS current will increase, leads to the enlargement of loss on coil winding. The increased loss is harmonic copper loss. In order to gain the loss, superposition principle is applied which is to consider every harmonics as independent power supply[8]. In the end, the total harmonic copper loss can be got. As to single phase transformer, the expression of harmonic copper loss is shown below.

$$
\Delta P_{n C u}=\sum_{n=2}^{\infty}\left(I_{n}^{2}(1) R_{n}(1)+I_{n}^{2}(2) R_{n}(2)\right) .
$$

Similarly, magnetic hysteresis loss and eddy-current loss will be bigger because of the harmonics, which make the increase of iron loss. The increased part can be reflected by the loss on excitation resistance. The harmonic iron loss is expressed as Eq. 8 accordingly.

$$
\Delta P_{n F e}=\sum_{n=2}^{\infty}\left(I_{n}^{2}(F e) R_{n}(F e)\right)
$$

So the total harmonic loss is:

$$
\Delta P_{n}=\sum_{n=2}^{\infty}\left(I_{n}^{2}(1) R_{n}(1)+I_{n}^{2}(2) R_{n}(2)+I_{n}^{2}(F e) R_{n}(F e)\right)
$$

Calculation of Harmonic Loss. It is easily found from Eq. 9 that the harmonic and harmonic parameters should be measured in order to get harmonic loss. Harmonic current could be detected by special devices. While as to the harmonic parameters, scholar from University of Clmenson made repeated short-circuit test and no-load test by controlling the order of harmonics, and parametric polynomial is achieved through mathematical fitting[9].

$$
R_{n}(1) / R_{1}(1)=0.96636081 e^{0.02685 n} .
$$


$X_{n}(1) / X_{1}(1)=0.9987219832+0.153923 \times 10^{-2} n-0.359525 \times 10^{-3} n^{2}$.

$R_{n}(2) / R_{1}(2)=0.96636081 e^{0.02685 n}$.

$X_{n}(2) / X_{1}(2)=0.9987219832+0.153923 \times 10^{-2} n-0.359525 \times 10^{-3} n^{2}$.

$R_{n}(\mathrm{Fe}) / R_{1}(\mathrm{Fe})=0.7060448503+0.248140685 n-0.26485 \times 10^{-2} n^{2}$.

$X_{n}(\mathrm{Fe}) / X_{1}(\mathrm{Fe})=1.077990695-0.0664264 n+0.119525 \times 10^{-2} n^{2}$.

$n$ is the order of harmonics.

Define $k_{n}(1)=k_{n}(2)=k_{n}=0.96636081 e^{0.02685 n}$. It's thus clear that harmonic resistance parameters is in direct proportion to fundamental harmonic resistance parameters. Therefore, the harmonic resistance when calculating copper loss can be expressed as Eq. 16.

$$
R_{n}=k_{n} R_{T}
$$

So the harmonic copper loss expression is shown below:

$$
\begin{aligned}
\Delta P_{n C u} & =3 \sum_{n=2}^{\infty} I_{n}^{2} R_{n} \\
& =3 I_{1}^{2} \sum_{n=2}^{\infty} \frac{I_{n}^{2}}{I_{1}^{2}} R_{n} \\
& =3 I_{1}^{2} R_{T} \sum_{n=2}^{\infty} k_{n} H R I_{n}^{2} \\
& =\Delta P_{C u} \sum_{n=2}^{\infty} k_{n} H R I_{n}^{2}
\end{aligned}
$$

$I_{1}$ is fundamental harmonic current, $H R I_{n}$ is every order of harmonic current.

Also define $k_{\mathrm{Fe}}=R_{n}(\mathrm{Fe}) / R_{1}(\mathrm{Fe})$. So the harmonic iron loss is deduced.

$$
\begin{aligned}
\Delta P_{n F e} & =\sum_{n=2}^{\infty} \frac{U_{n}^{2}}{R_{n}} \\
& =\frac{U_{1}^{2}}{R_{1}(F e)} \sum_{n=2}^{\infty} \frac{U_{n}^{2}}{k_{F e} U_{1}^{2}} . \\
& =\Delta P_{F e} \sum_{n=2}^{\infty} \frac{1}{k_{F e}} H R U_{n}^{2}
\end{aligned}
$$

$U_{1}$ is fundamental harmonic voltage, $H R U_{n}$ every order of harmonic voltage.

According to the analysis, the total harmonic loss can be expressed considering the harmonic effect. 


$$
\begin{aligned}
\Delta P_{\text {total }}= & \Delta P_{C u}\left(1+\sum_{n=2}^{\infty} k_{n} H R I_{n}^{2}\right) \\
& +\Delta P_{F e}\left(1+\sum_{n=2}^{\infty} \frac{1}{k_{F e}} H R U_{n}^{2}\right)
\end{aligned} .
$$

\section{Design of Comprehensive Loss Meter of Distribution Transformer}

Principle of Loss Meter. On the basis of former analysis, loss of fundamental harmonic parameter and loss of different harmonic parameter should be considered separately in order to get the total loss. Thus, firstly measure to get the one side real time running data with PT and CT. Then obtain the fundamental harmonic and harmonic component by calculation. According to the calculative process of copper loss and iron loss, fundamental harmonic total loss by Eq. 6; and the harmonic loss can be got based on the harmonic copper loss and harmonic iron loss of different order. After that, the total harmonic loss is got through Eq. 19. At last, add the two parts of losses to gain the total loss of transformer. The integrated principle of loss meter designed in this article in shown in Fig.2.

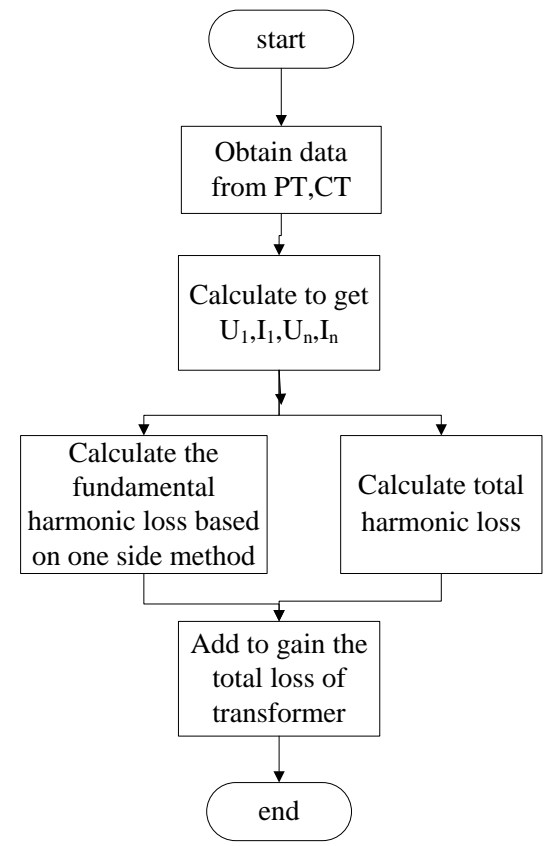

Fig. 2. The integrated principle of loss meter

Design of Hardware Circuit. 1) CT/PT. CT/PT is used to get the voltage and current data from transformers. In order to make the meter has widely application, the precision demand of CT/PT is comparatively high. Considering the common three-phase transformer, the three-phase voltage and current value can be got through two CT/PT. Current transformer LA58-P and voltage transformer CLSM-10MA are used in this meter.

2) Conditioning circuit. Operational amplifier constitute the voltage and current follower to condition the original voltage and current signal $I_{a}, I_{b}, U_{b a}, U_{c b}$, make it adapt to the input of ADC.

3) ADC. The four signal after conditioning will be converted to digital signal by MAX1324. The ADC module has multi-channel signal, but 4 channel are used, the rest connected to ground. The output precision is 14 bits.

4) DSP2812.2812 is the core module of the whole circuit, which is used to process and operate the signals to get the final result. Firstly, the input signals based on $I_{c}=-\left(I_{a}+I_{b}\right)$ and $U_{c a}=U_{b a}+U_{c b}$ to obtain three-phase voltage and current value. Then calculate the harmonics of different orders by FFT. So the fundamental harmonic loss and harmonic loss can be got through Eq. 6 and Eq. 9. In the same time, add to gain the total loss of transformer. 
5) Communication module. Zigbee is applied to receive data from DSP, and transmit to the display screen and database for observation and storage.

The main hardware circuits according to the preceding text analysis are shown below:

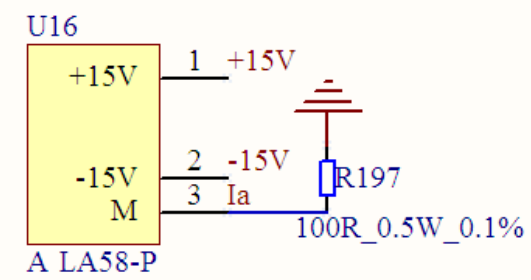

Fig. 3. Sampling circuit

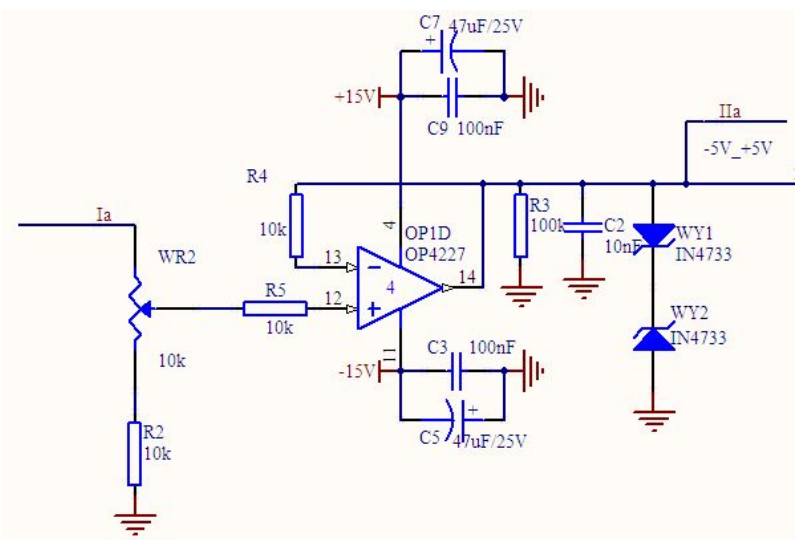

Fig. 4. Sampled signal conditioning circuit

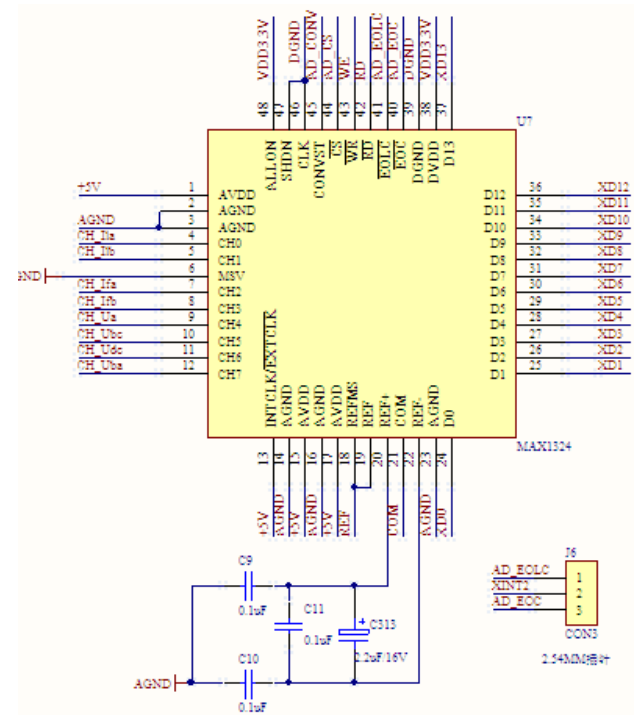

Fig. 5. ADC circuit

\section{Summary}

In power system, with the continuous appearance and application of non-linear load, lots of harmonics produced which leads to influence to transformer and higher the loss. The loss meter designed in this article also consider the loss caused by harmonics, and can get a comparatively precise result after appropriate simplification. Since using the one side measurement idea instead of two sides method, measurement devices only need to be installed on one side of the transformer, which highly extends the application range, guarantee the precision and decrease risk of operation. It has a good application prospect in the development of energy saving, cost reducing and energy efficiency increase. 


\section{References}

[1] WU Xi-hong, Research on the on-line detection method for the loss and capacity of distribution transformer, D. Chongqing. Chongqing University. (2006).

[2] HAN Shao-zong, Study on energy saving of accelerate weeping out high loss distribution transformer, J. Urban Construction Theory Research. 17 (2011).

[3] LIN Huai-de, ZHANG Yong-jun, HE Tong, Benefit evaluation of updating high energy-consumption distribution transformer, J. Power Demand Side Management. 12 (2010) 45-48.

[4] Wang Yan-liang Yue Zhi-shun Wang Jin-quan Zhang Qi, Analyzing the addition loss of harmonic and how to reducing it for energy-saving, J. Electrical Engineering. 2 (2009) 7.

[5] LIU Cheng-jun,YANG Ren-gang, Calculation and analysis of transformer's harmonic loss, J. Power System Protection and Control. 36 (2008) 33-36.

[6] SHI Bi-wei, Analysis of harmonic loss and terminal problems on transformer, D. Beijing. North China Electric Power University. (2014).

[7] GUO Hong-bo,TAN Jun-yuan, Effects of harmonics on transformer and its suppression measures, J. Electric Power Construction. 29 (2008) 35-37.

[8] ZHANG Zhan-long, WANG Ke, LI De-wen, et al, Transformer harmonic loss calculation and influence factor analysis, J. Power System Protection and Control. 39 (2011) 68-72.

[9] MENG Qin-liang, The analysis of effect on electricity price in transformer under the condition of harmonic, D. Baoding. North China Electric Power University. (2003). 